\title{
8 Herausforderungen einer klimafreundlichen Energieversorgung
}

\author{
Janine Kleemann, Kirsten Neumann, Antje Zehm
}

Sucht man nachhaltige Lösungen zur Begrenzung des globalen, anthropogenen Temperaturanstiegs, ist eine kritische Auseinandersetzung mit der Energieversorgung unablässig. In Deutschland wurde in einem ersten Schritt der Energiewende der Ausbau erneuerbarer Energien massiv forciert. Es zeigt sich, dass mit einer Vielzahl ausgereifter Technologien die Versorgungssicherheit gewährleistet werden kann.

Um eine Vorstellung davon zu bekommen, welche enormen Veränderungen die Energiewende hin zur Nutzung nachhaltiger Energieträger mit sich bringt, lohnt sich der Blick auf den aktuellen Energieverbrauch. Dabei wird zwischen Primär-, Endbeziehungsweise Nutzenergieverbrauch unterschieden. Unter dem Primärenergieverbrauch versteht man in der Energiewirtschaft jene Energiemenge, die durch die originären Energieträger (fossil, atomar oder erneuerbar) bereitgestellt und verbraucht wird, bevor eine technische Umwandlung erfolgt. Durch Umwandlung dieser Energieträger in technischen Anlagen sowie die Verteilung kommt es zu Verlusten. Die resultierenden Energieströme werden als Sekundärenergie (nach der technischen Umwandlung) oder Endenergie (bei den Endverbraucher:innen) bezeichnet. Zieht man davon schließlich die Verluste ab, die bei den Endverbraucher:innen entstehen, wie etwa Wärmeverluste einer Glühbirne, erhält man die Größe der Nutzenergie.

\section{Energieverbrauch - ein Faktencheck}

Der weltweite Primärenergieverbrauch lag im Jahr 2018 bei rund 590 Exajoule (EJ) ${ }^{52}$ und setzte sich aus der Nutzung von Erdöl (circa 34 Prozent), Kohle (circa 27 Prozent), Erdgas (circa 24 Prozent) sowie Kernenergie und erneuerbaren Energien (jeweils circa 4 Prozent) zusammen (Breitkopf 2019). Die Zahlen zur Entwicklung des weltweiten Primärenergieverbrauchs steigen seit 1990 kontinuierlich an, von 1990 bis 2017 um rund 58 Prozent. Den größten Anteil am Primärenergiebedarf haben China (2017: circa 22 Prozent), die Vereinigten Staaten von Amerika (2017: circa 25 Prozent) und Europa (2017: circa 12 Prozent) (IEA 2020).

52 Exajoule (1 EJ $=10^{18}$ Joule). 
Aus den Zahlen für Europa geht hervor, dass mit etwa 58 EJ im Jahr 2018 der Gesamtprimärenergieverbrauch im Vergleich zum Vorjahr um 0,71 Prozent gesunken ist, während der Endenergieverbrauch minimal im Vergleich zum Vorjahr anstieg (plus 0,02 Prozent) (Eurostat 2020). Zwölf EU-Mitgliedsstaaten weisen eine Zunahme des Primärenergieverbrauchs auf (größter Anstieg: Estland, plus 9 Prozent), während elf Staaten ihren Primärenergieverbrauch reduzieren konnten (größter Rückgang: Belgien, minus 5 Prozent). Positiv zu bewerten ist der weiterhin steigende Anteil an erneuerbaren Energien (Windkraft, Solarenergie, Wasserkraft, Biokraftstoffe) um circa 5 Prozent bezogen auf das Referenzjahr 2011, bezogen auf das Referenzjahr 1990 sogar um 12 Prozent.

In Deutschland leisten heute neben den fossilen Energieträgern Erdgas, Kohle und Erdöl erneuerbare Energieträger einen stetig steigenden Beitrag zur Deckung des Energiebedarfs. Insgesamt wurden im Jahr 2019 in Deutschland 12,8 EJ Primärenergie verbraucht, wobei eine rückläufige Tendenz feststellbar ist $(2,1$ Prozent im Vergleich zum Vorjahr) (AGEB e. V. 2020). Etwa ein Drittel dieser Energiemenge wird in Deutschland generiert, die weiteren zwei Drittel werden durch Importe abgedeckt. Kohle, Öl und Gas hatten einen Anteil von 78 Prozent am Primärenergiebedarf, der Anteil der Kernenergie betrug 6 Prozent, während die erneuerbaren Energien 15 Prozent des Primärenergieverbrauchs ausmachten. Vergleicht man diesen Wert der erneuerbaren Energien mit ihrem Anteil im Jahr 1990, zeigt sich eine signifikante Steigerung um 14 Prozent (UBA 2020). Der Endenergieverbrauch betrug im Jahr 2018 circa 9,0 EJ. Abb. 8.1 zeigt die Aufteilung auf die verschiedenen Energieträger: Es dominieren Kraftstoffe/Mineralöle (37 Prozent), Gase (24 Prozent) und Strom ${ }^{53}$ (21 Prozent).

Der Endenergieverbrauch Deutschlands ist gegenüber 1990 nur leicht rückläufig (UBA 2020). Trotz steigender Energieeffizienz und der Einsparung von Energie in zahlreichen Anwendungen ist - bedingt durch Wirtschaftswachstum und Steigerung des Konsums - keine signifikante Reduktion des Endenergieverbrauchs festzustellen. Ein genauer Einblick lässt sich anhand einer Aufteilung des Endenergieverbrauchs auf die Sektoren Verkehr, Industrie, Gewerbe/Handel/Dienstleistungen sowie Privathaushalte gewinnen. Der Verkehr war der verbrauchsintensivste Sektor, mehr als 90 Prozent der Kraftstoffe stammen aus fossilen Energieträgern (Mineralölen). Zudem weist der Verkehrssektor seit 2010 fast durchgängig einen Anstieg des Endenergieverbrauchs auf. Grund dafür ist das zunehmende Verkehrsaufkommen auf der Straße, sowohl im Personen- als auch im Güterverkehr. Technische Effizienzsteigerungen in den Fahrzeugen konnten den Anstieg des Endenergieverbrauchs nicht kompensieren. Im industriellen Sektor ist demgegenüber ein Rückgang des Endener-

53 Einschließlich des Stroms, der mit erneuerbaren Energien erzeugt wurde. 
Primärenergieverbrauch

Deutschland 2018 (Gesamt: 13,1 EJ)

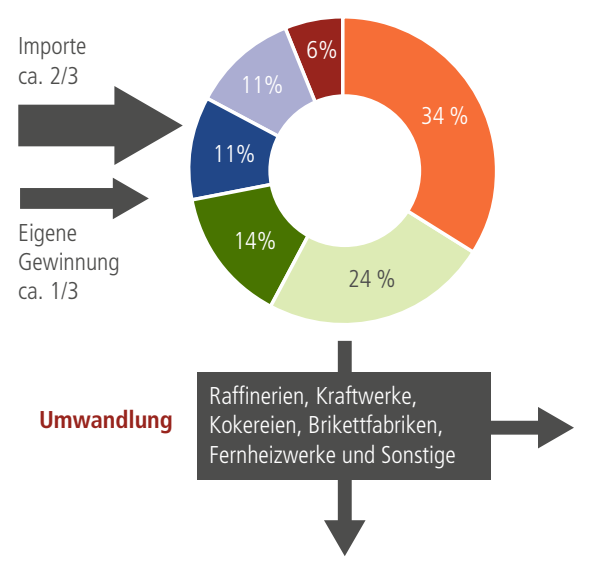

Endenergieverbrauch Deutschland 2018

(Gesamt: 9,0 EJ)

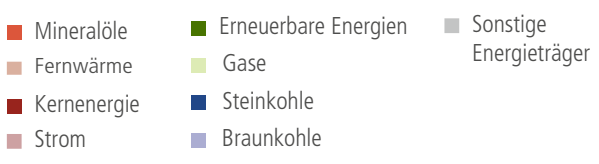

- Verbrauch und Verluste im Umwandlungsbereich; Fackel- und Leistungsverluste (3,3 EJ)

- Ausfuhr, Hochseebunkerungen,

Bestandsaufstockungen $(4,4 \mathrm{EJ})$

- Nichtenergetischer Verbrauch $(0,8 \mathrm{EJ})$
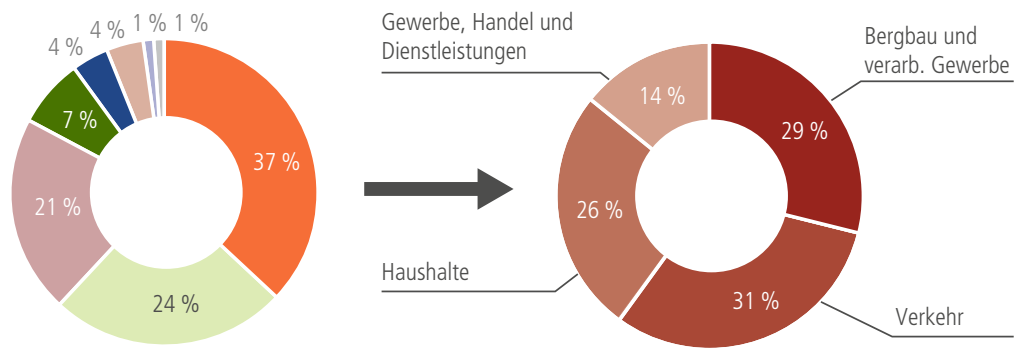

Abb. 8.1 Primär- und Endenergieverbrauch in Deutschland 2018 mit Umwandlungsverlusten und Endenergieverbrauch nach Sektoren, Grafik modifiziert aus Energieflussbildern. (Eigene Darstellung nach AGEB e. V. 2020)

gieverbrauchs (2,3 Prozent von 2017 zu 2018) zu verzeichnen, hier schlagen Fortschritte in der Energieeffizienz zu Buche (Destatis 2019). Bezogen auf die Nutzenergie werden in der Industrie zwei Drittel der Energie als Prozesswärme aufgerufen, ein Viertel als mechanische Energie (AGEB e. V. 2020). Auch im Sektor Privathaushalte ist ein leichter Rückgang des Endenergiebedarfs zu verzeichnen. Die Nutzenergiebilanz zeigt im Detail, dass hier drei Viertel der Energie zur Erzeugung von Raumwärme aufgewendet wird. Auch im Sektor Gewerbe, Handel, Dienstleistungen ist Raumwärme mit mehr als 50 Prozent der entscheidende Verbraucher. 


\section{Energiewende: Chancen und Herausforderungen}

Um dem Klimawandel zu begegnen, hat die Bundesregierung geplant, die deutschen Treibhausgasemissionen gegenüber 1990 stetig zu reduzieren (um 35 Prozent bis 2020, um 40 Prozent bis 2030, um 70 Prozent bis 2040 und um 80 bis 95 Prozent bis 2050). Der vorliegende Green-Deal-Entwurf der EU-Kommission geht sogar weit darüber hinaus und sieht ein treibhausgasneutrales Europa bis 2050 vor (siehe auch Kap. 4 „European Green Deal: Hebel für internationale Klima- und Wirtschaftsallianzen"). Der Umgang mit und die Sichtweise auf Energie wird sich also ändern müssen, sollen die Ziele erreicht werden. So wird man in einer treibhausgasneutralen Zukunft nicht mehr in der Kategorie "Technologie" denken, sondern in der Kategorie "Funktionalität". Der Fokus verlagert sich auch in der Energieversorgung weg vom Energieträger hin zum Energieservice (Wärme, Strom, Mobilität) und dessen Bereitstellung.

\section{Energiesparpotenziale}

Der auf den ersten Blick einfachste und wirtschaftlichste Weg, die Treibhausgasemissionen zu senken, besteht darin, Energie einzusparen. Für den Verkehrssektor sieht der European Green Deal für eine treibhausgasneutrale Energieversorgung Europas Einsparungen von 90 Prozent vor (EU Kommission 2019). Diese Quote entspricht dem Anteil, welcher gegenwärtig durch fossile Brennstoffe gedeckt wird, sodass der Verkehrssektor nach dem aktuellen Stand auf alle Fahrzeuge mit Verbrennungsmotoren verzichten müsste. Vor dem Hintergrund, dass der Energiebedarf in diesem Sektor in Deutschland aktuell sogar zunimmt, ist die Erarbeitung politischer Maßnahmen für eine Verkehrswende unausweichlich und überaus dringend (siehe Kap. 7 "Auf dem Weg zu einer nachhaltigen Mobilität").

In den Sektoren Industrie und Privathaushalte wird Energie überwiegend für Wärme aufgewendet, sodass hier verstärkt Energieeinsparpotenziale identifiziert und genutzt werden müssen, mit dem Ziel, eine "Wärmewende" zu realisieren. Die vorgeschriebenen Standards für Neubauten liegen mit aktuell etwa 55 Kilowattstunden pro Quadratmeter und Jahr (KfW100-Standard) im Bereich dessen, was mit erneuerbaren Energien wie Solarenergie, Erdwärme oder durch Nahwärme ${ }^{54}$ aufgebracht werden kann. Da im Gebäudealtbestand der Verbrauch besonders hoch ist, sollten mittelfristig Neubauten nur noch als Null- oder Plusenergiehäuser genehmigt werden, die in der Bilanz keine Energie benötigen bzw. sogar einen Überschuss erzeugen. Aktuell werden zwar Niedrigenergiehäuser gefördert, jedoch endet die Staf-

${ }^{54}$ Abwärmenutzung zum Beispiel aus Rechenzentren, Industriebetrieben oder Mini-Blockheizkraftwerken. 
felung bei $\mathrm{KfW} 40^{55}$, sodass ein Plusenergiehaus keine höhere Förderung erhält als ein KfW40-Haus. Die im Nationalen Aktionsplan Energieeffizienz angedachten Einsparungen durch Sanierungen im Gebäudebestand setzen auf Freiwilligkeit. Bei besonders ineffizienten Gebäuden sollte jedoch zur Erreichung der Einsparziele geprüft werden, ob diese durch verpflichtende Maßnahmen zu ersetzen sind. Im Einzelfall wäre weiterhin zu prüfen, wann Sanierungen einem Abriss und Neubau vorzuziehen sind - auch vor dem Hintergrund, dass Beton ein sehr energieintensiver Baustoff ist.

\section{Ausbau Erneuerbarer Energien}

Im Zuge der Energiewende hat Deutschland sich gegenüber der Europäischen Union verpflichtet, den Anteil erneuerbarer Energien am Bruttoendenergieverbrauch bis 2020 auf 18 Prozent zu erhöhen (Bundesregierung 2020). Die politischen Ziele hinsichtlich des Anteils erneuerbarer Energien in der Stromversorgung sind bereits von der Realität überholt, da das Ziel von 40 bis 45 Prozent erneuerbarer Energien in der Stromversorgung bis zum Jahr 2025 bereits 2019 erreicht wurde. Im Hinblick auf den schnell fortschreitenden Klimawandel sollte deshalb der für Deutschland beschlossene Zeitplan zur Energiewende unter Einbeziehung der europäischen Zielsetzung überarbeitet und gestrafft werden.

Treibhausgasneutrale Energieerzeugung muss die Energiegewinnung aus fossilen Energieträgern durch erneuerbare Energiequellen wie Wind, Sonne, Erdwärme, Wasserkraft und Biogase ersetzen. Ein Weg, fossile Energieträger für eine $\mathrm{CO}_{2}$-neutrale Energiegewinnung zu nutzen, ist die Kernenergie. Problematisch sind jedoch die Risiken der Uranspaltung, welche zuletzt beim Kraftwerksunglück von Fukushima im März 2011 sichtbar wurden und in dessen Folge Deutschland den Atomausstieg beschloss. Im europäischen Ausland wurden im selben Zug die Vorschriften zum Betrieb neuer Atomkraftwerke verschärft, sodass der Bau neuer Anlagen unwirtschaftlich wird ${ }^{56}$. Ungelöst für alle Kernspaltungstechnologien - insbesondere auch für die Idee eines Thorium-Reaktors - ist die Entsorgung der radioaktiven Zerfallsprodukte. Perspektivisch kann Kernfusion eine Alternative darstellen. Um die dafür nötigen extrem hohen Temperaturen, ähnlich dem Inneren der Sonne, zu erreichen, ist ein enormer technischer Aufwand nötig, welcher jedoch in aktuellen Forschungsreaktoren grundsätzlich als bewältigt gilt. Der erste Kraftwerksreaktor zur Energieversorgung ITER ${ }^{57}$

${ }_{55}$ Der KfW40-Standard entspricht dem auf 40 Prozent des KfW100-Standards reduzierten Energieverbrauch.

56 Wie geschehen im Fall des geplanten Reaktorblocks IV des Kraftwerks Olkiluoto/Finnland, dessen Baupläne gestoppt wurden, weil die Hersteller die Fertigstellung nicht mehr sicherstellen können.

57 Lat. für "Weg". 


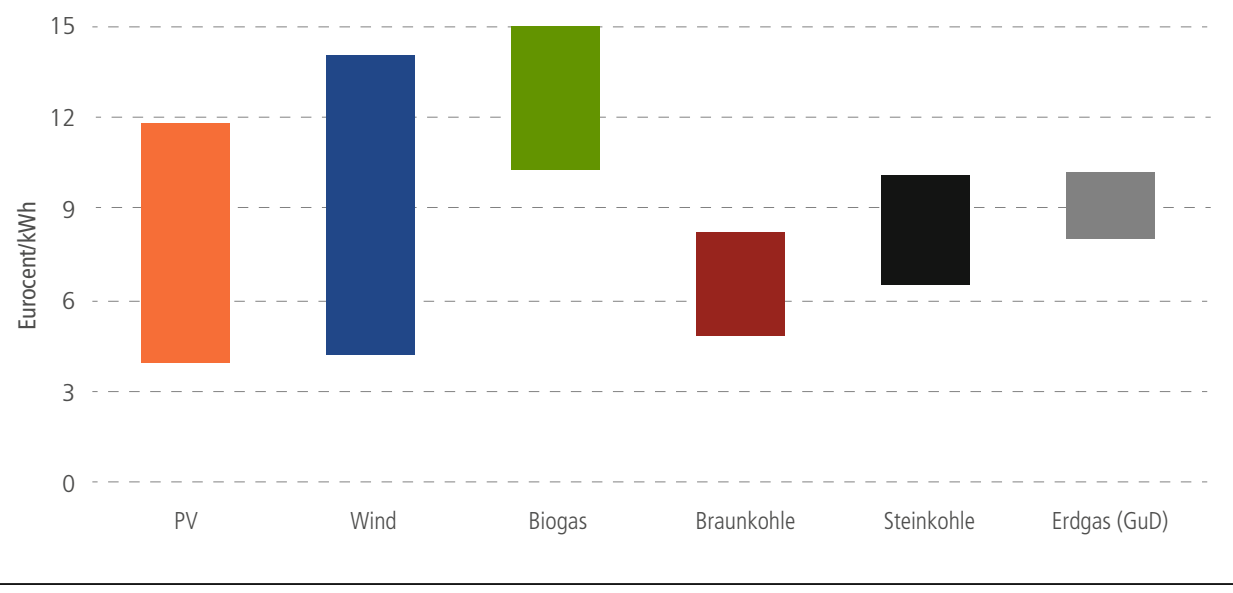

Abb. 8.2 Stromgestehungskosten in Eurocent pro Kilowattstunde ( $k$ Wh) für die erneuerbaren und fossilen Energieträger Photovoltaik (PV), Wind, Biogas, Braun- und Steinkohle, Erdgas in Form von kombinierten Gas- und Dampfkraftwerken (GuD). (Eigene modifizierte Darstellung nach Fh-ISE 2020)

entsteht aktuell in Südfrankreich. Er soll 2025 erste Fusionen ermöglichen und 2035 sein volles Leistungspotenzial erreichen (ITER 2020). Im Gegensatz zur Kernspaltung hat die Kernfusion den Vorteil, dass weder die Gefahr einer ungebremsten Reaktion besteht noch langfristig radioaktiver Abfall entsteht, sodass sie - sofern verfügbar ebenfalls als nachhaltige Energieform gelten und berücksichtigt werden kann.

Eine Umstellung auf erneuerbare Energieerzeugung ist aus wirtschaftlicher Sicht günstig. Abb. 8.2 zeigt eine Übersicht zu den Gestehungskosten für einzelne Energieträger. Zwar erreichen moderne fossile Kraftwerke Bruttowirkungsgrade ${ }^{58}$ zwischen 39 Prozent in modernen Kohlekraftwerken und 47 Prozent in Gaskraftwerken (UBA 2020), allerdings erzeugen moderne Photovoltaik- und Windenergieanlagen an günstigen Standorten bereits seit Jahren Strom zu günstigeren Gestehungskosten (Fh-ISE 2018). Eine weitere Kostendegression ist für Wind und Solarstrom zu erwarten, während die Gestehungskosten für Kohle und Gas aufgrund der steigenden Preise für $\mathrm{CO}_{2}$-Zertifikate steigen. Als Quelle großer Mengen an $\mathrm{CO}_{2}$-Emissionen ist somit die Fortführung des Betriebs insbesondere alter fossiler Kraftwerke kaum zu rechtfertigen.

Die Investition in Photovoltaikanlagen kann sich für Endverbraucher ohne Umlagebefreiung insbesondere in Kombination mit stationären Batteriespeichern sehr schnell

58 Der Bruttowirkungsgrad berücksichtigt nicht den Eigenverbrauch der Kraftwerke. 
rentieren. Folglich steigen die Ausbauzahlen stetig: Aktuell sind Photovoltaikanlagen mit einer Spitzenleistung von rund 60 Gigawatt (GW) installiert. Das gesamte Ausbaupotenzial vorhandener Gebäudeflächen in sinnvoller Ausrichtung wurde in einer aktuellen Studie auf 800 GW geschätzt (Fh-ISE 2020).

Dass Windenergie längst wirtschaftlich ist, zeigt sich darin, dass Energieversorgungsunternehmen beginnen, große Offshore-Windparks auch ohne staatliche Förderung zu bauen (Handelsblatt 2019). An Land sind Windparks noch günstiger zu errichten. Aktuelle Studien zu den Möglichkeiten zur Nutzung der Windenergie an Land sind schwer zu finden, es ist jedoch davon auszugehen, dass mehr Potenzial vorhanden ist als für die Energiewende benötigt. Eine Studie des Umweltbundesamtes aus dem Jahr 2013 identifizierte in Deutschland ein Potenzial von 1228 GW Leistung für Windparks an Land (UBA 2013). Unter Berücksichtigung der Entwicklungen zum sogenannten Repowering, also dem Austausch alter Anlagen durch neue, effizientere Anlagen, ist außerdem davon auszugehen, dass tatsächlich aus heutiger Sicht eine sehr viel höhere Leistung möglich ist. Insgesamt wird für die Umstellung des Energiesystems ein Vielfaches der bisher installierten Leistung aus erneuerbaren Quellen (circa $125 \mathrm{GW}$ ) benötigt. Schätzungen hängen stark davon $a b$, wie viel Treibhausgasemissionen noch erlaubt sind. Beispielsweise wurde der Bedarf an installierter Leistung aus Sonne und Wind für eine Reduktion der Treibhausgase um 80 bis 90 Prozent bis zum Jahr 2050 auf 290 bis 540 GW geschätzt (Fh-ISE 2015). Auf diese Weise könnten etwa 50 bis 60 Prozent des Primärenergieaufkommens gedeckt werden.

Die Gestehungskosten für Biogas können aktuell nicht mit denen für fossiles Gas konkurrieren, sodass hier weiterhin Förderanreize notwendig sein werden. Auch ist die Nutzung biogenen Materials zur Verwendung in Biogasanlagen begrenzt, da die Konkurrenz zu Nahrungs- und Energiepflanzen zu vermeiden ist.

Wasser- beziehungsweise Fließkraftwerke sind zur Produktion nachhaltiger Energie eine seit vielen Jahrzehnten etablierte Technologie, deren Potenzial in Deutschland allerdings schon zu mehr als 80 Prozent ausgeschöpft ist. Die Stromgestehungskosten liegen zwischen 5 Eurocent pro Kilowattstunde für Großanlagen und bis zu 20 Eurocent pro Kilowattstunde für kleinere Anlagen (BMWi 2015). Das verbleibende Potenzial kann durch Modernisierungen oder die Reaktivierung von Kraftwerken an bestehenden Stauhaltungen nutzbar gemacht werden.

Auch weltweit gewinnen erneuerbare Energien zunehmend an Relevanz. Im Jahr 2018 wurden weltweit für erneuerbare Energien 65 Prozent an Kapazitäten zugebaut und damit mehr als für fossile Energien (REN21 2020). 2019 wurden weltweit 363,3 Milliarden US-Dollar in saubere Energielösungen investiert (BloombergNEF 2020). Nach einer Studie des Bundesumweltministeriums (BMU) steigt das globale Marktvolumen im Bereich „Umweltfreundliche Erzeugung, Speicherung und Vertei- 
lung von Energie" von 667 Milliarden Euro im Jahr 2016 auf 1164 Milliarden Euro im Jahr 2025 (BMU 2018). Angesichts dieses Wachstumsmarktes kann sich eine schnelle Energiewende für Deutschland auch wirtschaftlich lohnen, indem die Unternehmen aufgrund ihres technologischen Know-hows und ihrer systemischen Lösungskompetenz international eine Vorreiterrolle einnehmen.

\section{Sektorenkopplung und Speicher}

Die Herausforderungen bei der Nutzung erneuerbarer Energieträger ergeben sich aus dem Umstand, dass Wind und Sonne nicht immer gleichbleibend verfügbar sind. Windenergie- und Photovoltaikanlagen erzeugen direkt Strom, sodass sich Wetteränderungen sofort im Stromnetz auswirken. Fossil betriebene Kraftwerke hingegen erzeugen kontinuierlich Strom mittels Dampfturbinen und stabilisieren so das Stromnetz. Da der Strom im Stromnetz nicht gespeichert werden kann, besteht die Herausforderung darin, den Stromverbrauch zu jeder Zeit auf die jeweils erzeugte Menge durch erneuerbare Energien abzustimmen. Der Verbrauch (Lastkurve) muss deshalb mittel- und langfristig weitgehend der fluktuierenden Erzeugungskurve folgen. Dafür muss einerseits der Verbrauch flexibilisiert werden. Gleichzeitig können Speicher und Sektorenkopplung (Power-to-X-Technologien) als Puffer wirken. Insbesondere Wärme und Mobilität werden mit dem Stromsystem verbunden werden müssen, wo sie als Speicher fungieren. Dies kann direkt durch die Nutzung von Strom im Verkehr mit der Bahn beziehungsweise in Elektroautos oder zum Betrieb von Wärmepumpen beziehungsweise Elektrodenkesseln zur Erzeugung von Wärme erfolgen. Pumpspeicherkraftwerke eignen sich für den Ausgleich tageszeitlicher bis wöchentlicher Schwankungen von Sonne und Wind, wobei Umwandlungsverluste in Kauf genommen werden müssen. Andererseits kann Strom auch indirekt etwa zur Herstellung synthetischer Brenn- und Kraftstoffe (Power-to-Gas oder Power-to-Liquid) wie Wasserstoff verwendet werden. Hierbei treten Verluste im Bereich von 30 Prozent pro Umwandlungsstufe auf.

Biokraftstoffe werden demnach nicht für die Stromproduktion an sich benötigt, sondern für diejenigen Anwendungen, welche sich nicht mit Strom bewerkstelligen lassen, wie Hochtemperaturanwendungen in der Industrie (zum Beispiel zur Stahlproduktion) oder als Kraftstoff für Containerschiffe oder Flugzeuge. Eine wichtige Option, um $\mathrm{CO}_{2}$ aus der Atmosphäre zu binden, ist die Methanisierung, bei der aus Wasserstoff unter Verwendung von $\mathrm{CO}_{2}$ synthetisches Methan erzeugt wird. Das in Deutschland gut ausgebaute Erdgasnetz bietet zusätzlich die Möglichkeit, das Gas zu transportieren und zu speichern. Aufgrund des enormen Speicherbedarfs strebt die Bundesregierung im Rahmen der Wasserstoffstrategie eine großflächige Installation von Anlagen zur Herstellung synthetischer Energieträger (Wasserstoff, Methan oder flüssige Brenn-/Kraftstoffe) an, um erneuerbare Brennstoffe zu erzeugen und diese bei Bedarf auch wieder zu verstromen. 
Zugleich gilt es, Maßnahmen zur Anpassung des Verbrauchs an die Stromerzeugung voranzutreiben. Dieser Zusammenhang wird über den Strommarkt geregelt: An der Strombörse in Leipzig wird viertelstundenweise Strom gehandelt. Dies erfolgt typischerweise am Spotmarkt bis zu einem Tag im Voraus, jedoch sind auch kurzfristig bis 30 Minuten vor der Lieferung noch Käufe am Intradaymarkt möglich. Dieses Strommarktdesign entspricht den Anforderungen eines Systems, in dem kontinuierlich Strom produziert wird. Bei zunehmender Volatilität ist jedoch eine Anpassung des Strommarktes notwendig, die eine erzeugungsgesteuerte Nutzung für Stromkund:innen ermöglicht. Alternativ dazu befinden sich vielversprechende Instrumente in praktischer Erprobung, mit denen sich Verbrauchskapazitäten ähnlich wie derzeit Strom handeln lassen (enera 2019). Zur Umsetzung solcher Maßnahmen und zur besseren Integration der Systeme gilt es, die Digitalisierung und die Verbreitung von Datensammelpunkten und Koppelstellen voranzutreiben.

\section{Das Stromnetz - Rückgrat der Energiewende}

Im Zuge einer treibhausgasneutralen Energieversorgung wird insbesondere der Energiebedarf der Sektoren Wärme und Verkehr zukünftig direkt oder indirekt (Powerto-Gas beziehungsweise Power-to-Liquid) mit Strom gedeckt werden müssen. Dies bedeutet nach aktuellen Schätzungen etwa eine Verdoppelung des Strombedarfs bis 2050 (acatech 2017). Demnach wird das Stromnetz für eine nachhaltige Energieversorgung in Zukunft erheblich mehr leisten müssen. Des Weiteren muss das Übertragungsnetz an die räumliche Verschiebung der Erzeugungsleistung von fossilen Kraftwerken in Süd- und Westdeutschland hin zu Offshore-Windanlagen in Norddeutschland angepasst werden, um Netzüberlastungen und damit das Risiko für Stromausfälle zu vermeiden. Gegenwärtig wird im Fall drohender Netzüberlast mit sogenannten Redispatch-Maßnahmen (Umplanung in den Kraftwerken) reagiert: meist durch Hochfahren lastnaher Kraftwerke oder das Herabregeln lastferner Erzeuger, typischerweise Windräder. So wurden 2020 im ersten Quartal 5,8TWh mit Redispatch-Maßnahmen im Netz verschoben und Grünstrom, hauptsächlich Wind, in Höhe von 3,0 TWh aufgrund überlasteter Netze abgeregelt (BNetzA 2020). Aus diesem Grund werden aktuell schneller Höchstspannungsleitungen gemäß dem Netzausbaubeschleunigungsgesetz von 2019 gebaut, wobei überwiegend neuartige Hochspannungs-Gleichstrom-Übertragungsleitungen (HGÜ) zum Einsatz kommen. Auch um die Investitionskosten im Rahmen zu halten, ist ein gezielter Ausbau der nachhaltigen Energieversorgung unvermeidbar.

Im Zuge der Liberalisierung der Strommärkte wurden 1998 in Deutschland die Netze wirtschaftlich von den Stromversorgungsunternehmen getrennt, um die Versorgung unabhängig vom Stromhandel sicherzustellen. Die Übertragungsnetzbetreiber (ÜNB: 50Hertz, TenneT, Amprion und TransnetBW) sind seither für die Fernleitungen im Be- 
reich der Höchstspannung zuständig und haben die Aufgabe, die eingespeisten und verbrauchten Energiemengen zu jeder Zeit ausgeglichen zu halten. Zu diesem Zweck kaufen sie der Situation entsprechend auch kurzfristig Strom am Regelenergiemarkt ein: Die Primärregelleistung, die sofort (für bis zu 15 Minuten) bereitgestellt werden muss, wird durch Kraftwerke (auch virtuelle) erbracht, die direkt vom Netzbetreiber gesteuert werden können. Sekundärregelleistung muss spätestens innerhalb von fünf Minuten abrufbar sein und wird ebenfalls automatisch vom Netzbetreiber gesteuert. Für die Minutenreserve, welche innerhalb einer Viertelstunde verfügbar sein muss, kommen Erzeugungseinheiten wie Pumpspeicherkraftwerke oder große, regelbare Lasten wie Stahl- oder Aluminiumwerke in Frage.

Im Zuge der Energiewende wird eine Steigerung der Regelenergiemengen notwendig sein: Mehr fluktuierende Erzeuger und Lasten im Stromnetz werden mehr Regelenergie erfordern, welche umso schneller bereitgestellt werden muss. Im Idealfall kann sich die Nachfrage durch steigende Preise selbst regeln. Die technischen und kommunikativen Anforderungen für Unternehmen, die flexible Energieleistungen anbieten, sind jedoch sehr hoch. Insbesondere die Kommunikation ist gerade bei dezentralen Anlagen ein Kostenfaktor, da ein Telefonanschluss „auf der grünen Wiese" oft nicht vorhanden und im ungünstigsten Fall auch keine Funkkommunikation möglich ist. In diesem Zusammenhang muss auch die strenge Auslegung der Entflechtungsregeln (Unbundling) für Stromanbieter, Netzbetreiber und Messstellenbetreiber kritisch betrachtet werden. Letztere haben am ehesten Zugang zu steuerbaren Lasten (über Netzanschluss und Zähler), dürfen jedoch keine Geschäftsmodelle zur Vermarktung der Flexibilität anbieten, auch wenn solche Lösungen technisch bereits erprobt werden.

Neben den Übertragungsnetzbetreibern gibt es ist Deutschland mehr als 900 Verteilnetzbetreiber für die Regionalversorgung im Bereich der Mittel- und Niederspannung. Mehr fluktuierende Energieerzeuger erfordern zusätzlich Verbesserungen in der Netzzustandserfassung insbesondere in der Mittelspannung, da aufgrund der kostspieligen Kommunikationsanbindung aktuell wenige Messstellen und automatisierte Schaltstellen vorhanden sind. Schaltvorgänge werden hier in der Regel per Telefon oder E-Mail anhand der Daten aus den Netzleitstellen ausgelöst. Die Automatisierung dieser Schalthandlungen ist im Rahmen einer Erweiterung der Leitsysteme in der Regel möglich, jedoch aufgrund der hohen Sicherheitsanforderungen auch kostspielig. Die Erfassung zusätzlicher Messdaten und die Möglichkeit einer automatisierten Steuerung sind außerdem Voraussetzung für den Einsatz von Netzoptimierungssoftware auch auf Basis von Methoden der Künstlichen Intelligenz etwa 
für das Blindleistungsmanagement ${ }^{59}$. Vor dem Hintergrund der Wirtschaftlichkeit wäre eine stärkere Zusammenarbeit zwischen Netzbetreibern und Kommunikationsanbietern wünschenswert, sie ist jedoch bisher eher selten, vermutlich aufgrund der großen Unterschiede in den Anforderungen an die Datensicherheit.

Auch die Steuerung in der Niederspannungsebene wird bei steigender Anzahl dezentraler Einspeiser und schwankender Last, wie etwa viele Ladevorgänge von Elektroautos, zunehmend zur Herausforderung. Hier ist in Ermangelung von Netzzustandsdaten so gut wie keine zentrale Steuerung „von oben" möglich. In diesem Zusammenhang wurde mit der Einführung der Smart Meter durch das Gesetz zur Digitalisierung der Energiewende 2015 das Ziel einer genaueren Erfassung von Netzzustandsdaten verfolgt, und zwar durch den Einbau digitaler Stromzähler mit Kommunikationsanbindung bei Einspeisern mit einer Einspeiseleistung über 7 Kilowatt (entsprechend einer größeren Solaranlage auf einer Scheune) oder bei größeren Verbrauchern oberhalb eines Jahresverbrauchs von 6000 Kilowattstunden. Der Einbau wird für die Messstellenbetreiber mit der Erklärung der Verfügbarkeit der Geräte im Januar 2020 nun innerhalb von acht Jahren verpflichtend. Praktisch hat sich gezeigt, dass die Kosten im Vergleich zum klassischen analogen, vor Ort ablesbaren Zähler oft hoch sind. Sie beinhalten die Entwicklung oder den Zukauf einer sehr sicheren Datenverschlüsselungssoftware, die Gerätekosten sowie die Installation der Kommunikationsanbindung. Kritisiert wird oft, dass der Nutzen der so ermittelten Daten für die Netzbetreiber aktuell gering ist, weil die Auslesezyklen nicht den sehr dynamischen Netzzustandsänderungen entsprechen und somit für die Netzführung kaum nennenswerte Hinweise geben. Eine Steuerung von Lasten oder Erzeugern, welche in den Geräten vorgesehen ist, ist - hauptsächlich wegen der Regeln zum Unbundling - derzeit nicht nutzbar. Dies sollte jedoch im Zuge der Netzdienlichkeit durch Angebote für die Regelleistung schnellstens ermöglicht werden.

Noch in der Entwicklung befinden sich Steuerungstechniken über sogenannte zelluläre Strukturen, wobei sich einzelne Netzabschnitte selbst regeln. Eine große Herausforderung ist dabei die Sicherheit von Daten sowie die Versorgungssicherheit. Die Autonomie derartiger Systeme reicht aktuell von netzversorgten Anlagen zur Optimierung der Stromkosten (wie bei einem Smarthome mit Solarstrom und einem Batteriespeicher) bis hin zu vollständig autonomen und isolierten Inselnetzen zur Selbstversorgung einzelner Einrichtungen. Wirtschaftlich getrieben durch den Wegfall der EEG-Umlage, bieten sie die Möglichkeit, die Versorgungssicherheit flexibel an die Bedürfnisse der Verbraucher:innen oder an veränderte Umgebungsbedingungen

59 Blindleistung, also nicht nutzbare Energieströme im Netz, die sich zwangsweise durch Transportverluste ergeben. Ein Blindleistungsmanagement optimiert also die im Netz transportierte Energie hinsichtlich der Transportverluste. 
anzupassen. Zudem haben Inselnetze größerer Industrieunternehmen das Potenzial, Teil der Lösung der Herausforderungen im Stromnetz insgesamt zu sein, indem sie das Versorgungsnetz entlasten. Auch für die Erzeugung und Speicherung von Biokraftstoffen und Wasserstoff werden sie an Bedeutung gewinnen.

Schließlich haben die im Zuge der Transformation der Energieversorgung zu implementierenden Technologien großen Einfluss auf die sogenannte Schwarzstartfähigkeit, also die Fähigkeit, nach einem Komplettausfall (Blackout) das Stromnetz in allen Spannungsebenen wieder aufzubauen. Übertragungs- und Verteilnetzbetreiber müssen für diesen Fall Konzepte zur Wiedererlangung der Stromversorgung vorhalten. Diese Konzepte können jedoch aufgrund der hohen Versorgungssicherheit schlecht erprobt werden und müssen gleichwohl ständig den Veränderungen im Netz durch zusätzliche Erzeugungseinheiten angepasst werden. Andererseits sind ErneuerbareEnergie(EE)-Anlagen wie Windräder viel besser als konventionelle Großkraftwerke in der Lage, nach einem Blackout wieder Strom zu liefern (der Wind weht weiter). Die Herausforderung liegt darin, die Spannungshaltung sicherzustellen, für die die EE-Anlagen per se nicht ausgestattet sind. In diesem Zusammenhang könnten Inselnetze, sofern sie für Notfälle schaltbar an das öffentliche Netz angeschlossen sind, die Schwarzstartfähigkeit der öffentlichen Netze unterstützen und eine Steuerung der Netze "von unten" anstoßen. Die damit verbundenen Herausforderungen lassen sich insgesamt den Fragen der Leistungselektronik für die Steuerung, der Kommunikationstechnik sowie der Entscheidungshoheit und der damit verbundenen Haftung (Autorität) zuordnen.

Aktuell wird in Deutschland etwa ein Fünftel der Energie über das Stromnetz bereitgestellt, wovon im April 2020 bezogen auf das Jahr etwa 56 Prozent, an manchen Tagen auch bis zu 80 Prozent, erneuerbaren Energien entstammt. Trotz des hohen Anteils volatiler erneuerbarer Energien ist die Versorgungssicherheit uneingeschränkt sehr hoch.

\section{Zusammenfassung und Ausblick}

Die Energiewende ist eine gesamtgesellschaftliche Aufgabe, zu deren Lösung eine Vielzahl ausgereifter Technologien zur Verfügung stehen, um die Versorgungssicherheit sicherzustellen. Die Herausforderung für die Politik und Wirtschaft besteht nun darin, die Transformation des Energiesystems hin zu einer $\mathrm{CO}_{2}$-neutralen Versorgung möglichst auch wirtschaftlich nachhaltig zu erreichen. Im Folgenden sind die wichtigsten, teilweise kurzfristig umzusetzenden Maßnahmen zusammengefasst: 
Senkung des Energieverbrauchs

- Maßnahmen zur Reduktion des Verkehrsaufkommens

- Förderung von verpflichtenden Energieeinsparmaßnahmen im Wärmebereich, zum Beispiel Nutzung von Abwärme und Solarthermie, Langzeitspeicherung von Wärme und Sanierungsmaßnahmen im Bestand

Nachhaltigkeit durch Umstellung auf erneuerbare Energieträger

- konsequenter Ausbau der Kapazitäten erneuerbarer Energien, insbesondere von Windenergie und Photovoltaik an den günstigsten Standorten

- Förderung der Sektorenkopplung durch Speicher: in erster Linie Batterien und Power-to-Heat, welche sehr effizient sind, aber auch Power-to-Gas zur Langzeitspeicherung und Versorgung nicht direkt elektrisch betreibbarer Verbraucher

- Reform des Strommarktes einerseits hinsichtlich eines größeren Handelsvolumens und andererseits hinsichtlich eines lokalen Handels von Strom und Lasten bis hin zum Stromkunden

- Reduktion der Anzahl von $\mathrm{CO}_{2}$-Zertifikaten

Versorgungssicherheit durch Stärkung der Netze

- Fortführung des Netzausbaus im Bereich der Höchstspannung

- Potenziale der Digitalisierung in der Energiewirtschaft heben, zum Beispiel durch Ausbau von Kommunikationsanbindungen sowie Energiemanagementsoftware

- Stärkung der Regelenergiekapazitäten: Überprüfung der Entflechtungsregeln zwischen Netzbetreiber und Stromanbieter in Sinne von Geschäftsmodellen, welche die Smart Meter tatsächlich "smart" machen

- Unterstützung des Aufbaus von Inselnetzen bzw. autonomen Netzabschnitten, zum Beispiel durch Förderung der benötigten Leistungselektronik

Die staatlichen Investitionen durch die Transformation der Netze werden dem wissenschaftlichen Beirat des BMWi zufolge auf rund 52 Milliarden Euro bis 2030 geschätzt (BMWi 2020). Hinzu kommen Investitionskosten für Speicher und die Dekarbonisierung der Industrie, welche hauptsächlich durch Wirtschaft und Privathaushalte getragen werden. Diese Aufwendungen sind notwendig, um zum einen den Weg hin zur klimaneutralen Energieversorgung in Deutschland zu meistern, aber auch, um die Vorreiterrolle Deutschlands im globalen Wettbewerb und den vorhandenen Technologievorsprung zu nutzen und auszubauen. Nur wenn die Energiewende in Deutschland und Europa gelingt, werden auch wirtschaftlich schwächere Regionen der Welt dieses Ziel anstreben. 


\section{Literatur}

acatech (2017): Deutsche Akademie der Technikwissenschaften: Sektorkopplung - Optionen für die nächste Phase der Energiewende - Kurzfassung der Stellungnahme. Online verfügbar unter www.acatech.de/publikation/sektorkopplung-optionen-fuer-die-naechstephase-der-energiewende/, zuletzt geprüft am 18.06.2020.

AGEB e. V. (2020): Aktuelle Daten zu Primärenergieverbrauch, Energieflussbilder, Bilanzen 1990-2018. Online verfügbar unter https://ag-energiebilanzen.de, zuletzt geprüft am 18.06.2020.

BloombergNEF (2020): Clean Energy Investment Trends, 2019. Online verfügbar unter https://data.bloomberglp.com/professional/sites/24/BloombergNEF-Clean-Energy-Investment-Trends-2019.pdf, zuletzt geprüft am 18.06.2020.

BMWi (2015): Bundesministerium für Wirtschaft und Energie: Marktanalyse Wasserkraft. Online verfügbar unter www.erneuerbare-energien.de/EE/Redaktion/DE/Downloads/ bmwi_de/marktanalysen-photovoltaik-wasserkraft.pdf, zuletzt geprüft am 18.06.2020.

BMWi (2020): Entwicklung und Stand der öffentlichen Investitionen und Qualität der Infrastruktur. Online verfügbar unter www.bmwi.de/Redaktion/DE/Publikationen/Ministerium/Neroeffentlichung-Wissenschaftlicher-Beirat/gutachten-oeffentliche-infrastruktur-indeutschland.pdf?_blob=publicationFile\&v=12, zuletzt geprüft am 04.08.2020.

BMU (2018): Bundesministerium für Umwelt, Naturschutz und nukleare Sicherheit: GreenTech made in Germany 2018 - Umwelttechnik-Atlas für Deutschland. Online verfügbar unter http://www.bmu.de/fileadmin/Daten_BMU/Pools/Broschueren/greentech_2018_ bf.pdf, zuletzt geprüft am 04.08.2020.

BNetzA (2020): Bundesnetzagentur: Bericht zu Netz- und Systemsicherheitsmaßnahmen - 1. Quartal 2019. Online verfügbar unter www.bundesnetzagentur.de/SharedDocs/Mediathek/Berichte/2020/Quartalszahlen_Q1_2020.pdf?_blob=publicationFile\&v=3, zuletzt geprüft am 22.09.2020.

Breitkopf, A. (2019): Weltweiter Primärenergieverbrauch in den Jahren von 1980 bis 2018. Online verfügbar unter https://de.statista.com/statistik/daten/studie/42226/umfrage/weltinsgesamt---verbrauch-an-primaerenergie-in-millionen-tonnen-oelaequivalent/, zuletzt geprüft am 18.06.2020.

Bundesregierung (2020): Aktuelle Zahlen zu Nachhaltigkeitspolitik und Energiewende. Bezahlbare und saubere Energie. Online verfügbar unter www.bundesregierung.de/bregde/themen/, zuletzt geprüft am 18.06.2020.

Destatis (2019): Pressemitteilung vom 20.12.2019: Energieverbrauch in der Industrie 2018um 2,3 Prozent gegenüber dem Vorjahr gesunken. Online verfügbar unter www. destatis.de/DE/Presse/Pressemitteilungen/2019/12/PD19_502_435.html, zuletzt geprüft am 18.06.2020.

enera (2019): SINTEG-Projekt enera: Die EnergiePlattform: Eine marktbasierte Lösung zum nachweislichen Handeln und Fernwirken regionaler grüner Energie. Online verfügbar 
unter www.sinteg.de/fileadmin/media/Publikationen/Jahreskonferenz_2019/Praesentationen/05_Session3_Heitmann_Onnen.pdf, zuletzt geprüft am 19.06.2020.

Europäische Kommission (2019): Mitteilung der Kommission an das Europäische Parlament, den Europäischen Rat, den Rat, den Europäischen Wirtschafts- und Sozialausschuss und den Ausschuss der Regionen. Online verfügbar unter https://eur-lex.europa.eu/resource. html?uri=cellar:b828d165-1c22-11ea-8c1f-01aa75ed71a1.0021.02/DOC_1\&format=PDF, zuletzt geprüft am 18.06.2020.

Eurostat (2020): Pressemitteilung vom 04.02.2020: Energieverbrauch im Jahr 2018. Online verfügbar unter https://ec.europa.eu/eurostat/documents/2995521/10341549/804022020-BP-DE.pdf/3e62b994-68fb-0ea8-7d29-f1769272bf5a, zuletzt geprüft am 18.06.2020.

Fh-ISE (2015): Fraunhofer-Institut für Solare Energiesysteme: Was kostet die Energiewende? Wege zur Transformation des deutschen Energiesystems bis 2050. Online verfügbar unter www.ise.fraunhofer.de/content/dam/ise/de/documents/publications/studies/FraunhoferISE-Studie-Was-kostet-die-Energiewende.pdf, zuletzt geprüft am 19.06.2020.

Fh-ISE (2018): Fraunhofer-Institut für Solare Energiesysteme: Stromgestehungskosten Erneuerbare Energien. Online verfügbar unter www.ise.fraunhofer.de/content/dam/ise/de/ documents/ publications/studies/DE2018_ISE_Studie_Stromgestehungskosten_Erneuerbare_Energien.pdf, zuletzt geprüft am 18.06.2020.

Fh-ISE (2020): Fraunhofer-Institut für Solare Energiesysteme: Aktuelle Fakten zur Photovoltaik in Deutschland. Online verfügbar unter www.ise.fraunhofer.de/content/dam/ise/de/documents/publications/studies/aktuelle-fakten-zur-photovoltaik-in-deutschland.pdf, zuletzt geprüft am 19.06.2020.

Handelsblatt (2019): Orsted baut ersten Windpark auf See ohne Fördergelder. Online verfügbar unter www.handelsblatt.com/unternehmen/energie/erneuerbare-orstedbaut-ersten-windpark-auf-see-ohne-foerdergelder/25296420.html?ticket=ST-755843Hant4jLq2iPj4fxYk0mB-ap4, zuletzt geprüft am 19.06.2020.

IEA (2020): International Energy Agency - Data and Statistics. Online verfügbar unter https:// www.iea.org/data-and-statistics?country=WORLD\&fuel=Energy\%20supply\&indicator=Coal\%20production\%20by\%20type, zuletzt geprüft am 18.06.2020.

ITER (2020): Aboout ITER. Online verfügbar unter www.iter.org, zuletzt geprüft am 19.06.2020.

REN21 (2020): Renewables 2019 - Global Status Report. Online verfügbar unter www. ren21.net/wp-content/uploads/2019/05/gsr_2019_full_report_en.pdf, zuletzt geprüft am 19.06.2020.

UBA (2013): Potenzial der Windenergie an Land. Online verfügbar unter www.umweltbundesamt.de/sites/default/files/medien/378/publikationen/potenzial_der_windenergie.pdf, zuletzt geprüft am 18.06.2020.

UBA (2020): Aktuelle Daten des Umweltbundesamtes u. a. zu Klima, Energie und Verkehr. Online verfügbar unter www.umweltbundesamt.de/daten, zuletzt geprüft am 18.06.2020. 


\section{(c) (1)}

Dieses Kapitel wird unter der Creative Commons Namensnennung 4.0 International Lizenz http://creativecommons.org/licenses/by/4.0/deed.de) veröffentlicht, welche die Nutzung, Vervielfältigung, Bearbeitung, Verbreitung und Wiedergabe in jeglichem Medium und Format erlaubt, sofern Sie den/die ursprünglichen Autor(en) und die Quelle ordnungsgemäß nennen, einen Link zur Creative Commons Lizenz beifügen und angeben, ob Änderungen vorgenommen wurden.

Die in diesem Kapitel enthaltenen Bilder und sonstiges Drittmaterial unterliegen ebenfalls der genannten Creative Commons Lizenz, sofern sich aus der Abbildungslegende nichts anderes ergibt. Sofern das betreffende Material nicht unter der genannten Creative Commons Lizenz steht und die betreffende Handlung nicht nach gesetzlichen Vorschriften erlaubt ist, ist für die oben aufgeführten Weiterverwendungen des Materials die Einwilligung des jeweiligen Rechteinhabers einzuholen. 